\title{
Lack of BV bounds for approximate solutions to a two-phase transition model arising from vehicular traffic
}

\author{
Mohamed Benyahia, Massimiliano D. Rosini
}

\begin{abstract}
We consider wave-front tracking approximate solutions to a two-phase transition model for vehicular traffic. We construct an explicit example showing that the total variation in space of the solution blows up in finite time even for an initial datum with bounded total variation.
\end{abstract}

2010 Mathematical Subject Classification. Primary: 35L65, 90B20, 35L45

Key words. Conservation laws, phase transitions, Lighthill-Whitham-Richards model, Aw-Rascle-Zhang model, point constraint on the density flux, wave-front tracking, blow up in finite time.

\section{Introduction}

In this paper we consider a two-phase transition model of hyperbolic conservation laws with point constraint on the density flux introduced in [20]. The application of such model is, for instance, the modelling of vehicular traffic along a road with pointlike inhomogeneities characterized by limited capacity, such as speed bumps, construction sites, toll gates, etc.

We distinguish two phases: the congested phase $\Omega_{\mathrm{c}}$ and the free-flow phase $\Omega_{\mathrm{f}}$. The model couples a $2 \times 2$ system of conservation laws for the congested phase, with a scalar conservation law for the free-flow phase. The coupling is achieved via phase transitions, namely discontinuities between two states belonging to different phases and satisfying the Rankine-Hugoniot conditions.

The first two-phase model has been proposed by Colombo [17]. Experimental data show that the density flux represented in the fundamental diagram is one-dimensional for high velocities, whereas it covers a twodimensional domain for low velocities, see [17, Figure 1.1]. For this reason it is reasonable to describe the traffic in the congested phase with a $2 \times 2$ system of conservation laws and the traffic in the free regime with a scalar conservation law.

Later on Goatin proposed in [26] a two-phase model obtained by coupling the ARZ model by Aw, Rascle and Zhang [5,37] for the congested phase $\Omega_{\mathrm{c}}$, with the LWR model by Lighthill, Whitham and Richards [31,34] for the free-flow phase $\Omega_{\mathrm{f}}$. Recall that this model has been recently generalized in [8].

Both the models introduced in [17] and [26] assume that $\Omega_{\mathrm{c}} \cap \Omega_{\mathrm{f}}=\emptyset$. The first two-phase model considering a metastable phase $\Omega_{\mathrm{c}} \cap \Omega_{\mathrm{f}} \neq \emptyset$ has been introduced in [11]. Notice that, differently from [8,26], for the models in $[11,17]$ the density flux vanishes at a unique maximal density, whose inverse corresponds to the average length of the vehicles. Here we do not consider a metastable phase and assume $\Omega_{\mathrm{c}} \cap \Omega_{\mathrm{f}}=\emptyset$. We also assume that $\Omega_{\mathrm{f}}$ is characterized by a unique value $V_{\mathrm{f}}$ for the velocity. At last we consider an heterogeneous traffic with vehicles having different lengths and allow the density flux to vanish at different densities.

These two-phase models have been recently generalized in $[9,20]$ by considering Riemann problems, namely Cauchy problems for piecewise constant initial data with a single jump, coupled with a point constraint on the density flux, so that at the interface $x=0$ the density flux of the solution must be lower than a given constant quantity $F \geqslant 0$. This condition is referred to as unilateral point constraint on the density flux, see [35] and the references therein. In vehicular traffic, a point constraint accounts for pointlike inhomogeneities of the road and models, for instance, the presence of a toll gate across which the flow of the vehicles cannot exceed its capacity $F$.

Existence results for unconstrained Cauchy problems for the above mentioned two-phase transition models have been proved in $[8,11,19,26]$. The first existence result for the constrained Cauchy problem has been established in [7] for the case with a metastable phase. In the present paper, we focus on the constrained Cauchy problem without a metastable phase. More precisely, as in [7] we use the Riemann solvers established in [8] and [20] in a wave-front tracking scheme to construct a sequence of approximate solutions $\left\{u_{n}\right\}_{n}$, which converges at the limit $n \rightarrow \infty$ to a solution $u$. We show that the total variation (in space) of $u_{n}$ becomes arbitrarily large as $n$ goes to infinity and that the total variation of solution $u$ blows up in finite time, even if the initial datum $u^{o}$ has bounded total variation. The construction of our example relies on the fact that the 
assumption $\Omega_{\mathrm{c}} \cap \Omega_{\mathrm{f}}=\emptyset$ allows to consider phase transitions from $\Omega_{\mathrm{c}}$ to $\Omega_{\mathrm{f}}$ and that the (density) flow of a non-classical shock can be lower than the maximal flow $F$ allowed by the constraint. We stress that both these properties do not occur in the case $\Omega_{\mathrm{c}} \cap \Omega_{\mathrm{f}} \neq \emptyset$ and this is exploited in [7]. Our example can be then useful to generalize the existence result proved in [7] to the case $\Omega_{\mathrm{c}} \cap \Omega_{\mathrm{f}}=\emptyset$ and to understand which conditions on the initial data guarantee existence of global-in-time solutions.

For completeness in the following we briefly recall some results for conservation laws. We firstly focus on the scalar case. Kruzhkov [29] established global well-posedness results in the class of so-called entropy solutions to Cauchy problems for scalar conservation laws with initial data in $\mathbf{L}^{\infty}$. Oleinik [33] proved that if the flux is $\mathbf{C}^{\mathbf{2}}$ and uniformly convex, then the solution has bounded total variation, even if the initial datum is only in $\mathbf{L}^{\infty}$. More recently, Ambrosio and De Lellis [2] improved Oleinik's result showing that the solution is a special function of bounded variation, at least except at most countably many times; we defer the reader to [3, §4] for the definition of special functions of bounded variation. Moreover by Schaeffer's regularity theorem [36] the solution has locally at most a finite number of discontinuity curves. This result is sharp in the sense that the regularity does not hold for every smooth initial datum, see the examples constructed in [36, §5] and [1].

About systems of conservation laws in one space dimension, the pioneering work by Glimm [24] established global in time existence of distributional solutions to Cauchy problems for strictly hyperbolic systems, with each characteristic field being either genuinely nonlinear or linearly degenerate and initial data having sufficiently small total variation. Uniqueness results were obtained by Bressan and several collaborators in a series of papers, we defer the reader to book [12]. Several regularity results that apply to scalar conservation laws with convex fluxes have been extended to systems of conservation laws with genuinely nonlinear vector fields, see for instance Glimm and Lax [25], Liu [32], Bressan and Colombo [15], and Bianchini and Caravenna [10].

As a matter of fact, a satisfactory well-posedness theory for hyperbolic systems of conservation laws is available only for data with small total variation. In the case of large data the well-posedness holds also as long as the total variation remains bounded $[14,30]$. The main remaining open problem is whether, for initial data with large total variation, the total variation remains uniformly bounded or can blow up in finite time. Examples of finite time blow up have been constructed in $[6,13,16,28]$.

The paper is organized as follows. In the next section we recall the two-phase transition model under consideration. In Section 3 we recall the Riemann solvers that are used then in the last section to construct our example.

\section{The model}

Before introducing model (2), we collect the parameters of the model together with their properties, see Figure 1.
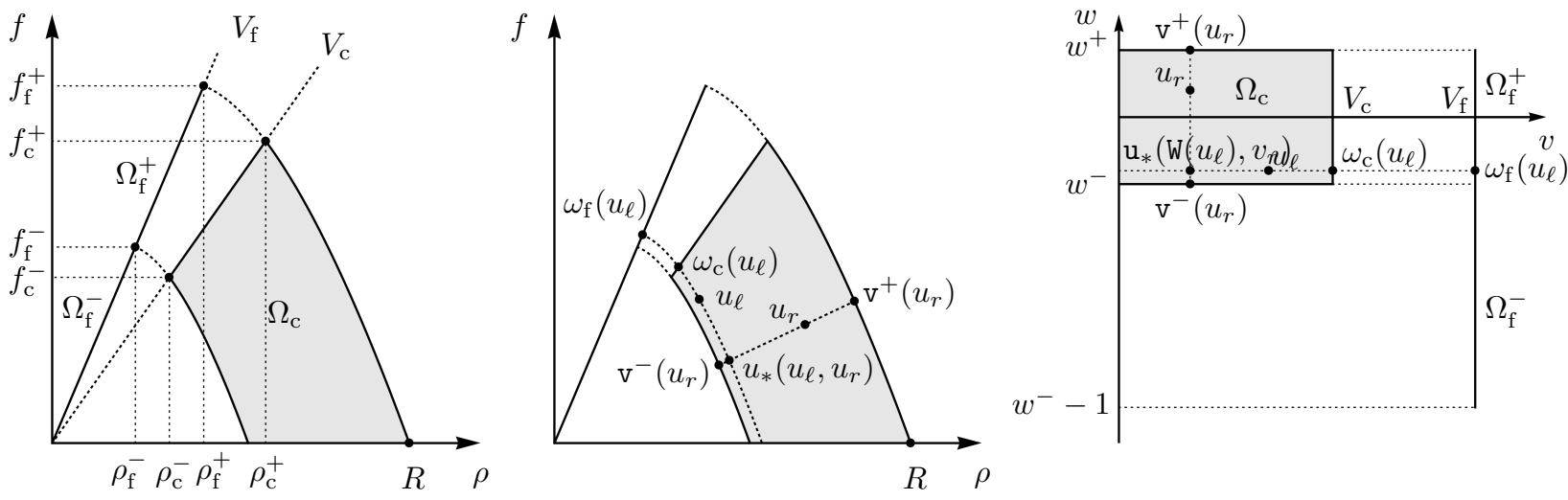

Figure 1: Parameters and notations.

Let $\rho \geqslant 0$ and $v \geqslant 0$ be the density and the velocity of the vehicles, respectively. Denote $u \doteq(\rho, v)$ and let $f(u) \doteq v \rho$ be the density flux. If $V_{\mathrm{f}}>0$ is the unique velocity in the free-flow phase $\Omega_{\mathrm{f}}$ and $\rho_{\mathrm{f}}^{+}$is the maximal density in $\Omega_{\mathrm{f}}$, then

$$
\Omega_{\mathrm{f}} \doteq\left\{u \in \mathbb{R}_{+}^{2}: \rho \leqslant \rho_{\mathrm{f}}^{+}, v=V_{\mathrm{f}}\right\},
$$

where $\mathbb{R}_{+} \doteq[0, \infty)$. If $V_{\mathrm{c}} \in\left(0, V_{\mathrm{f}}\right)$ is the maximal velocity in the congested phase $\Omega_{\mathrm{c}}$ and it is reached for densities ranging in $\left[\rho_{\mathrm{c}}^{-}, \rho_{\mathrm{c}}^{+}\right]$with $0<\rho_{\mathrm{c}}^{-}<\rho_{\mathrm{c}}^{+}$, then

$$
\Omega_{\mathrm{c}} \doteq\left\{u \in \mathbb{R}_{+}^{2}: v \leqslant V_{\mathrm{c}}, w^{-} \leqslant v+p(\rho) \leqslant w^{+}\right\},
$$


where $w^{ \pm} \doteq p\left(\rho_{\mathrm{c}}^{ \pm}\right)+V_{\mathrm{c}}$. Above $p \in \mathbf{C}^{2}((0, \infty) ; \mathbb{R})$ is an anticipation factor, which takes into account drivers' reactions to the state of traffic in front of them, see [5]. We assume that

$$
p^{\prime}(\rho)>0, \quad 2 p^{\prime}(\rho)+p^{\prime \prime}(\rho) \rho>0 \quad \text { for every } \rho>0 .
$$

Typical choices for $p$ are $p(\rho) \doteq \rho^{\gamma}$ with $\gamma>V_{\mathrm{f}} /\left(w^{-}-V_{\mathrm{f}}\right)>0$, or $p(\rho) \doteq V_{\text {ref }} \ln \left(\rho / \rho_{\max }\right)$ with $V_{\text {ref }}>V_{\mathrm{f}}$ and $\rho_{\max }>0$, see $[5,26]$.

Assume that $w^{+}=p\left(\rho_{\mathrm{f}}^{+}\right)+V_{\mathrm{f}}$ and that $\rho_{\mathrm{f}}^{-} \doteq p^{-1}\left(w^{-}-V_{\mathrm{f}}\right)$ is well defined. Let $R \doteq p^{-1}\left(w^{+}\right)>0$ be the maximal density (in the congested phase). Introduce

$$
f_{\mathrm{f}}^{ \pm} \doteq V_{\mathrm{f}} \rho_{\mathrm{f}}^{ \pm}, \quad f_{\mathrm{c}}^{ \pm} \doteq V_{\mathrm{c}} \rho_{\mathrm{c}}^{ \pm},
$$

and assume that $f_{\mathrm{c}}^{+} \in\left(f_{\mathrm{f}}^{-}, f_{\mathrm{f}}^{+}\right)$, so that

$$
0<f_{\mathrm{c}}^{-}<f_{\mathrm{f}}^{-}<f_{\mathrm{c}}^{+}<f_{\mathrm{f}}^{+} .
$$

Define

$$
\begin{array}{lll}
\Omega \doteq \Omega_{\mathrm{f}} \cup \Omega_{\mathrm{c}}, & \Omega_{\mathrm{f}}^{-} \doteq\left\{u \in \Omega_{\mathrm{f}}: \rho \in\left[0, \rho_{\mathrm{f}}^{-}\right)\right\}, & \Omega_{\mathrm{c}}^{\mathrm{ex}} \doteq\left\{u \in \mathbb{R}_{+}^{2}: v \leqslant V_{\mathrm{f}}, w^{-} \leqslant v+p(\rho) \leqslant w^{+}\right\} \\
& \Omega_{\mathrm{f}}^{+} \doteq\left\{u \in \Omega_{\mathrm{f}}: \rho \in\left[\rho_{\mathrm{f}}^{-}, \rho_{\mathrm{f}}^{+}\right]\right\}, & \Omega^{\mathrm{ex}} \doteq \Omega_{\mathrm{f}}^{-} \cup \Omega_{\mathrm{c}}^{\mathrm{ex}}
\end{array}
$$

Notice that $\Omega_{\mathrm{c}}^{\mathrm{ex}} \cap \Omega_{\mathrm{f}}=\Omega_{\mathrm{f}}^{+}$. We assume that

$$
v<p^{\prime}(\rho) \rho \text { for every }(\rho, v) \in \Omega_{\mathrm{c}}^{\mathrm{ex}} .
$$

Let the (extended) Lagrangian marker w: $\Omega^{\mathrm{ex}} \rightarrow\left[w^{-}-1, w^{+}\right]$and $\mathrm{W}: \Omega^{\mathrm{ex}} \rightarrow\left[w^{-}, w^{+}\right]$be defined by

$$
\mathrm{W}(u) \doteq\left\{\begin{array}{ll}
v+p(\rho) & \text { if } u \in \Omega_{\mathrm{c}}^{\mathrm{ex}}, \\
w^{-}-1+\frac{\rho}{\rho_{\mathrm{f}}^{-}} & \text {if } u \in \Omega_{\mathrm{f}}^{-},
\end{array} \quad \mathrm{W}(u) \doteq \max \left\{w^{-}, \mathrm{w}(u)\right\} .\right.
$$

The constrained PT model is then represented by the constrained Cauchy problem for

$$
\begin{aligned}
& \text { Free-flow (LWR) } \\
& \text { Congested flow (ARZ) } \\
& \left\{\begin{array}{l}
u=(\rho, v) \in \Omega_{\mathrm{f}}, \\
\rho_{t}+\left(\rho V_{\mathrm{f}}\right)_{x}=0, \\
v=V_{\mathrm{f}},
\end{array}\right. \\
& \left\{\begin{array}{l}
u=(\rho, v) \in \Omega_{\mathrm{c}}, \\
\rho_{t}+(\rho v)_{x}=0, \\
(\rho \mathrm{W}(u))_{t}+(\rho \mathrm{W}(u) v)_{x}=0,
\end{array}\right.
\end{aligned}
$$

with initial condition

$$
u(0, x)=u^{o}(x),
$$

and point constraint on the density flux at $x=0$

$$
f\left(u\left(t, 0_{ \pm}\right)\right) \leqslant F
$$

where $F \geqslant 0$ is a given constant quantity.

In Section 4 we consider PT model (2) with $F \in\left(f_{\mathrm{c}}^{+}, f_{\mathrm{f}}^{+}\right)$and choose an initial datum $u^{o}$ with bounded total variation such that the total variation of the corresponding solution $u$ blows up in finite time. The solution $u$ is obtained as the limit of approximate solutions $u_{n}$ constructed by applying a wave-front tracking algorithm analogous to that used in [7], see [12,27] and the references therein. Our wave-front tracking algorithm relies on the Riemann solvers $\mathcal{R}, \mathcal{R}_{F}: \Omega^{2} \rightarrow \mathbf{B V}(\mathbb{R} ; \Omega)$ for the unconstrained and constrained Riemann problems for (2). For completeness we recall their definitions in the next section.

\section{The Riemann solvers}

In this section we give the definitions of the Riemann solvers $\mathcal{R}_{\mathrm{ARZ}}: \Omega_{\mathrm{c}}^{2} \rightarrow \mathbf{B V}\left(\mathbb{R} ; \Omega_{\mathrm{c}}\right), \mathcal{R}, \mathcal{R}_{F}: \Omega^{2} \rightarrow \mathbf{B V}(\mathbb{R} ; \Omega)$ for the ARZ model and for the PT models without or with point constraint, respectively defined in $[5,8,20]$. We recall that in general a Riemann solver $\mathcal{R S}: \mathcal{D}^{2} \rightarrow \mathbf{B V}(\mathbb{R} ; \mathcal{D})$ gives the solution corresponding to any Riemann problem, namely to any Cauchy problem with initial datum

$$
u^{o}(x)= \begin{cases}u_{\ell} & \text { if } x<0 \\ u_{r} & \text { if } x \geqslant 0\end{cases}
$$


with $u_{\ell}, u_{r} \in \mathcal{D}$, by letting

$$
u(t, x) \doteq \mathcal{R S}\left[u_{\ell}, u_{r}\right](x / t) .
$$

We introduce the following functions, see Figure 1:

$$
\begin{array}{ll}
\mathrm{r}_{\mathrm{f}}:\left[w^{-}, w^{+}\right] \rightarrow\left[\rho_{\mathrm{f}}^{-}, \rho_{\mathrm{f}}^{+}\right], & \mathrm{r}_{\mathrm{f}}(w) \doteq p^{-1}\left(w-V_{\mathrm{f}}\right), \\
\mathrm{r}_{\mathrm{c}}:\left[w^{-}, w^{+}\right] \rightarrow\left[\rho_{\mathrm{c}}^{-}, \rho_{\mathrm{c}}^{+}\right], & \mathrm{r}_{\mathrm{c}}(w) \doteq p^{-1}\left(w-V_{\mathrm{c}}\right), \\
\omega_{\mathrm{f}}:\left[w^{-}, w^{+}\right] \rightarrow \Omega_{\mathrm{f}}^{+}, & \omega_{\mathrm{f}}(w) \doteq\left(\mathrm{r}_{\mathrm{f}}(w), V_{\mathrm{f}}\right), \\
\omega_{\mathrm{c}}:\left[w^{-}, w^{+}\right] \rightarrow \Omega_{\mathrm{c}}, & \omega_{\mathrm{c}}(w) \doteq\left(\mathrm{r}_{\mathrm{c}}(w), V_{\mathrm{c}}\right), \\
\mathrm{v}^{ \pm}:\left[0, V_{\mathrm{c}}\right] \rightarrow \Omega_{\mathrm{c}}, & \mathrm{v}^{ \pm}(v) \doteq\left(p^{-1}\left(w^{ \pm}-v\right), v\right), \\
\mathrm{u}_{*} \doteq\left(\mathrm{r}_{*}, \mathrm{v}_{*}\right):\left[w^{-}, w^{+}\right] \times\left[0, V_{\mathrm{c}}\right] \rightarrow \Omega_{\mathrm{c}}, & \mathrm{u}_{*}(w, v) \doteq\left(p^{-1}(w-v), v\right), \\
\Lambda:\left\{\left(u_{\ell}, u_{r}\right) \in \Omega^{2}: \rho_{\ell} \neq \rho_{r}\right\} \rightarrow \mathbb{R}, & \Lambda\left(u_{\ell}, u_{r}\right) \doteq \frac{f\left(u_{r}\right)-f\left(u_{\ell}\right)}{\rho_{r}-\rho_{\ell}} .
\end{array}
$$

For any $w \in\left[w^{-}, w^{+}\right]$, let

$$
\lambda_{w}:\left[\mathrm{r}_{\mathrm{f}}(w), p^{-1}(w)\right] \rightarrow\left[-p^{-1}(w) p^{\prime}\left(p^{-1}(w)\right), V_{\mathrm{f}}-\mathrm{r}_{\mathrm{f}}(w) p^{\prime}\left(\mathrm{r}_{\mathrm{f}}(w)\right)\right]
$$

be defined by $\lambda_{w}(\rho) \doteq w-p(\rho)-\rho p^{\prime}(\rho)$ and let

$$
\mathcal{R}_{w}:\left[-p^{-1}(w) p^{\prime}\left(p^{-1}(w)\right), V_{\mathrm{f}}-\mathrm{r}_{\mathrm{f}}(w) p^{\prime}\left(\mathrm{r}_{\mathrm{f}}(w)\right)\right] \rightarrow\left[\mathrm{r}_{\mathrm{f}}(w), p^{-1}(w)\right]
$$

be its inverse function. Define $[a]_{+} \doteq \max \{a, 0\}$.

Definition 3.1. The Riemann solver $\mathcal{R}_{\mathrm{ARZ}}: \Omega_{\mathrm{c}}^{\mathrm{ex}} \times \Omega_{\mathrm{c}}^{\mathrm{ex}} \rightarrow \mathbf{B V}\left(\mathbb{R} ; \Omega_{\mathrm{c}}\right)$ for $A R Z$ model (2a) right, (2b), (3) is defined as follows:

(ARZ.1) If $u_{\ell}, u_{r} \in \Omega_{\mathrm{c}}^{\mathrm{ex}}$ with $\mathrm{w}\left(u_{\ell}\right)=\mathrm{w}\left(u_{r}\right)$ and $v_{r}<v_{\ell}$, then

$$
\mathcal{R}_{\mathrm{ARZ}}\left[u_{\ell}, u_{r}\right](\nu) \doteq \begin{cases}u_{\ell} & \text { if } \nu<\Lambda\left(u_{\ell}, u_{r}\right), \\ u_{r} & \text { if } \nu \geqslant \Lambda\left(u_{\ell}, u_{r}\right)\end{cases}
$$

(ARZ.2) If $u_{\ell}, u_{r} \in \Omega_{\mathrm{c}}^{\mathrm{ex}}$ with $\mathrm{w}\left(u_{\ell}\right)=\mathrm{w}\left(u_{r}\right)$ and $v_{\ell}<v_{r}$, then

$$
\mathcal{R}_{\mathrm{ARZ}}\left[u_{\ell}, u_{r}\right](\nu) \doteq \begin{cases}u_{\ell} & \text { if } \nu<\lambda_{\mathrm{W}\left(u_{\ell, r}\right)}\left(\rho_{\ell}\right), \\ \mathcal{R}_{\mathrm{w}\left(u_{\ell}\right)}(\nu) & \text { if } \lambda_{\mathrm{w}\left(u_{\ell, r}\right)}\left(\rho_{\ell}\right) \leqslant \nu<\lambda_{\mathrm{w}\left(u_{\ell, r}\right)}\left(\rho_{r}\right), \\ u_{r} & \text { if } \nu \geqslant \lambda_{\mathrm{w}\left(u_{\ell, r}\right)}\left(\rho_{r}\right) .\end{cases}
$$

(ARZ.3) If $u_{\ell}, u_{r} \in \Omega_{\mathrm{c}}^{\mathrm{ex}}$ with $\mathrm{w}\left(u_{\ell}\right) \neq \mathrm{w}\left(u_{r}\right)$ and $v_{\ell}=v_{r}$, then

$$
\mathcal{R}_{\mathrm{ARZ}}\left[u_{\ell}, u_{r}\right](\nu) \doteq \begin{cases}u_{\ell} & \text { if } \nu<v_{\ell, r} \\ u_{r} & \text { if } \nu \geqslant v_{\ell, r}\end{cases}
$$

(ARZ.4) If $u_{\ell}, u_{r} \in \Omega_{\mathrm{c}}^{\mathrm{ex}}$ with $\mathrm{w}\left(u_{\ell}\right) \neq \mathrm{w}\left(u_{r}\right)$ and $v_{r}<v_{\ell}$, then

$$
\mathcal{R}_{\mathrm{ARZ}}\left[u_{\ell}, u_{r}\right](\nu) \doteq \begin{cases}u_{\ell} & \text { if } \nu<\Lambda\left(u_{\ell}, \mathrm{u}_{*}\left(\mathrm{w}\left(u_{\ell}\right), v_{r}\right)\right), \\ \mathrm{u}_{*}\left(\mathrm{w}\left(u_{\ell}\right), v_{r}\right) & \text { if } \Lambda\left(u_{\ell}, \mathrm{u}_{*}\left(\mathrm{w}\left(u_{\ell}\right), v_{r}\right)\right) \leqslant \nu<v_{r} \\ u_{r} & \text { if } \nu \geqslant v_{r}\end{cases}
$$

$(A R Z .5)$ If $u_{\ell}, u_{r} \in \Omega_{\mathrm{c}}^{\mathrm{ex}}$ with $\mathrm{w}\left(u_{\ell}\right) \neq \mathrm{w}\left(u_{r}\right)$ and $v_{\ell}<v_{r}$, then

$$
\mathcal{R}_{\mathrm{ARZ}}\left[u_{\ell}, u_{r}\right](\nu) \doteq \begin{cases}u_{\ell} & \text { if } \nu<\lambda_{\mathrm{w}\left(u_{\ell}\right)}\left(\rho_{\ell}\right), \\ \mathcal{R}_{\mathrm{w}\left(u_{\ell}\right)}(\nu) & \text { if } \lambda_{\mathrm{w}\left(u_{\ell}\right)}\left(\rho_{\ell}\right) \leqslant \nu<\lambda_{\mathrm{w}\left(u_{\ell}\right)}\left(\mathrm{r}_{*}\left(u_{\ell}, u_{r}\right)\right), \\ \mathrm{u}_{*}\left(\mathrm{w}\left(u_{\ell}\right), v_{r}\right) & \text { if } \lambda_{\mathrm{w}\left(u_{\ell}\right)}\left(\mathrm{r}_{*}\left(u_{\ell}, u_{r}\right)\right) \leqslant \nu<v_{r}, \\ u_{r} & \text { if } \nu \geqslant v_{r} .\end{cases}
$$

(ARZ.6) If $u_{\ell}=u_{r} \in \Omega_{\mathrm{c}}^{\mathrm{ex}}$, then $\mathcal{R}_{\mathrm{ARZ}}\left[u_{\ell}, u_{r}\right] \doteq u_{\ell, r}$. 
Some comments on the above definition are in order. In case (ARZ.1) we have $\mathrm{r}_{\mathrm{f}}\left(\mathrm{w}\left(u_{\ell, r}\right)\right) \leqslant \rho_{\ell}<\rho_{r} \leqslant$ $p^{-1}\left(\mathrm{w}\left(u_{\ell, r}\right)\right)$ and $\mathcal{R}_{\mathrm{ARZ}}\left[u_{\ell}, u_{r}\right]$ has only the 1 -shock $\mathfrak{S}_{1}\left(u_{\ell}, u_{r}\right)$. In case (ARZ.2) we have $\mathrm{r}_{\mathrm{f}}\left(\mathrm{w}\left(u_{\ell, r}\right)\right) \leqslant \rho_{r}<$ $\rho_{\ell} \leqslant p^{-1}\left(\mathrm{w}\left(u_{\ell, r}\right)\right)$ and $\mathcal{R}_{\mathrm{ARZ}}\left[u_{\ell}, u_{r}\right]$ has only the 1-rarefaction $\mathfrak{R}_{1}\left(u_{\ell}, u_{r}\right)$. In case (ARZ.3) we have $v_{\ell}=v_{r}$ and $\mathcal{R}_{\mathrm{ARZ}}\left[u_{\ell}, u_{r}\right]$ has only the 2-contact discontinuity $\mathfrak{C}_{2}\left(u_{\ell}, u_{r}\right)$. In case (ARZ.4) we have $\mathrm{r}_{\mathrm{f}}\left(\mathrm{w}\left(u_{\ell, r}\right)\right) \leqslant \rho_{\ell}<$ $\mathbf{r}_{*}\left(u_{\ell}, u_{r}\right) \leqslant p^{-1}\left(\mathrm{w}\left(u_{\ell}\right)\right)$ and $\mathcal{R}_{\mathrm{ARZ}}\left[u_{\ell}, u_{r}\right]$ is the juxtaposition of $\mathfrak{S}_{1}\left(u_{\ell}, \mathrm{u}_{*}\left(u_{\ell}, u_{r}\right)\right)$ and $\mathfrak{C}_{2}\left(\mathrm{u}_{*}\left(u_{\ell}, u_{r}\right), u_{r}\right)$. In case (ARZ.5) we have $\mathrm{r}_{\mathrm{f}}\left(\mathrm{w}\left(u_{\ell, r}\right)\right) \leqslant \mathrm{r}_{*}\left(u_{\ell}, u_{r}\right)<\rho_{\ell} \leqslant p^{-1}\left(\mathrm{w}\left(u_{\ell}\right)\right)$ and $\mathcal{R}_{\mathrm{ARZ}}\left[u_{\ell}, u_{r}\right]$ is the juxtaposition of $\mathfrak{R}_{1}\left(u_{\ell}, \mathrm{u}_{*}\left(u_{\ell}, u_{r}\right)\right)$ and $\mathfrak{C}_{2}\left(\mathrm{u}_{*}\left(u_{\ell}, u_{r}\right), u_{r}\right)$.

Definition 3.2. The Riemann solver $\mathcal{R}_{\mathrm{PT}}: \Omega^{2} \rightarrow \mathbf{B V}(\mathbb{R} ; \Omega)$ for PT model (2a), (2b), (3) is defined as follows: (PT.1) If $u_{\ell}, u_{r} \in \Omega_{\mathrm{f}}$, then

$$
\mathcal{R}_{\mathrm{PT}}\left[u_{\ell}, u_{r}\right](\nu) \doteq \begin{cases}u_{\ell} & \text { if } \nu<\Lambda\left(u_{\ell}, u_{r}\right), \\ u_{r} & \text { if } \nu \geqslant \Lambda\left(u_{\ell}, u_{r}\right) .\end{cases}
$$

(PT.2) If $u_{\ell}, u_{r} \in \Omega_{\mathrm{c}}$, then $\mathcal{R}_{\mathrm{PT}}\left[u_{\ell}, u_{r}\right] \doteq \mathcal{R}_{\mathrm{ARZ}}\left[u_{\ell}, u_{r}\right]$.

(PT.3) If $u_{\ell} \in \Omega_{\mathrm{c}}$ and $u_{r} \in \Omega_{\mathrm{f}}$, then

$$
\mathcal{R}_{\mathrm{PT}}\left[u_{\ell}, u_{r}\right](\nu) \doteq \begin{cases}\mathcal{R}_{\mathrm{ARZ}}\left[u_{\ell}, \omega_{\mathrm{c}}\left(\mathrm{w}\left(u_{\ell}\right)\right)\right](\nu) & \text { if } \nu<\Lambda\left(\omega_{\mathrm{c}}\left(\mathrm{w}\left(u_{\ell}\right)\right), \omega_{\mathrm{f}}\left(\mathrm{w}\left(u_{\ell}\right)\right)\right), \\ \omega_{\mathrm{f}}\left(u_{\ell}\right) & \text { if } \Lambda\left(\omega_{\mathrm{c}}\left(\mathrm{w}\left(u_{\ell}\right)\right), \omega_{\mathrm{f}}\left(\mathrm{w}\left(u_{\ell}\right)\right)\right) \leqslant \nu<V_{\mathrm{f}}, \\ u_{r} & \text { if } \nu \geqslant V_{\mathrm{f}} .\end{cases}
$$

(PT.4) If $u_{\ell} \in \Omega_{\mathrm{f}}^{-}$and $u_{r} \in \Omega_{\mathrm{c}}$, then

$$
\mathcal{R}_{\mathrm{PT}}\left[u_{\ell}, u_{r}\right](\nu) \doteq \begin{cases}u_{\ell} & \text { if } \nu<\Lambda\left(u_{\ell}, \mathrm{v}^{-}\left(v_{r}\right)\right), \\ \mathcal{R}_{\mathrm{ARZ}}\left[\mathrm{v}^{-}\left(v_{r}\right), u_{r}\right](\nu) & \text { if } \nu \geqslant \Lambda\left(u_{\ell}, \mathrm{v}^{-}\left(v_{r}\right)\right) .\end{cases}
$$

(PT.5) If $u_{\ell} \in \Omega_{\mathrm{f}}^{+}$and $u_{r} \in \Omega_{\mathrm{c}}$, then $\mathcal{R}_{\mathrm{PT}}\left[u_{\ell}, u_{r}\right] \doteq \mathcal{R}_{\mathrm{ARZ}}\left[u_{\ell}, u_{r}\right]$.

We underline that $\mathcal{R}_{\mathrm{PT}}\left[u_{\ell}, u_{r}\right]$ has a phase transition in case (PT.3) between $\omega_{\mathrm{c}}\left(\mathrm{w}\left(u_{\ell}\right)\right)$ and $\omega_{\mathrm{f}}\left(\mathrm{w}\left(u_{\ell}\right)\right)$, in case (PT.4) between $u_{\ell}$ and $\mathrm{v}^{-}\left(v_{r}\right)$, and in case (PT.5) between $u_{\ell}$ and $\mathrm{u}_{*}\left(\mathrm{w}\left(u_{\ell}\right), v_{r}\right)$.

Fix $F \in\left(f_{\mathrm{c}}^{+}, f_{\mathrm{f}}^{+}\right)$. Since $(t, x) \mapsto \mathcal{R}_{\mathrm{PT}}\left[u_{\ell}, u_{r}\right](x / t)$ does not in general satisfy constraint condition (2c), we introduce

$$
\begin{aligned}
& \mathcal{D}_{1} \doteq\left\{\left(u_{\ell}, u_{r}\right) \in \Omega^{2}: f\left(\mathcal{R}_{\mathrm{PT}}\left[u_{\ell}, u_{r}\right]\left(t, 0_{ \pm}\right)\right) \leqslant F\right\}, \\
& \mathcal{D}_{2} \doteq \Omega^{2} \backslash \mathcal{D}_{1}=\left\{\left(u_{\ell}, u_{r}\right) \in \Omega^{2}: \mathrm{w}\left(u_{\ell}\right)>w_{F}, u_{r} \in \Omega_{\mathrm{f}}\right\},
\end{aligned}
$$

where $w_{F} \doteq \mathrm{w}\left(u_{F}\right) \in\left(w^{-}, w^{+}\right)$with $u_{F} \doteq\left(F / V_{\mathrm{f}}, V_{\mathrm{f}}\right) \in \Omega_{\mathrm{f}}^{+}$.

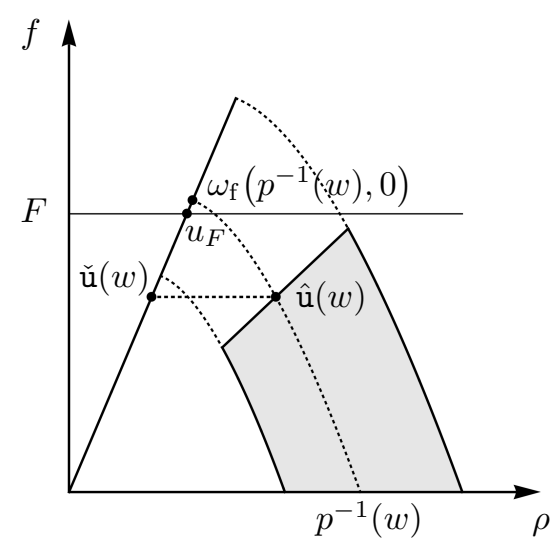

Figure 2: Geometrical meaning of $\hat{u}$ and $\check{u}$ defined in (4).

Define

$$
\begin{aligned}
\hat{\mathrm{u}}:\left(w_{F}, w^{+}\right] & \rightarrow \Omega_{\mathrm{c}}, \\
w & \mapsto \hat{\mathrm{u}}(w) \doteq \omega_{\mathrm{c}}(w), \\
\check{\mathrm{u}}:\left(w_{F}, w^{+}\right] & \rightarrow \Omega_{\mathrm{f}}, \\
w & \mapsto \check{\mathrm{u}}(w) \doteq\left(\frac{f(\hat{\mathrm{u}}(w))}{V_{\mathrm{f}}}, V_{\mathrm{f}}\right),
\end{aligned}
$$


see Figure 2. Notice that for any $w \in\left(w_{F}, w^{+}\right]$

$$
f(\check{\mathrm{u}}(w))=f(\hat{\mathrm{u}}(w))<F .
$$

Definition 3.3. The constrained Riemann solver $\mathcal{R}_{F}: \Omega^{2} \rightarrow \mathbf{B V}(\mathbb{R} ; \Omega)$ for constrained PT model (2) is defined as follows:

$$
\mathcal{R}_{F}\left[u_{\ell}, u_{r}\right](x) \doteq \begin{cases}\mathcal{R}_{\mathrm{PT}}\left[u_{\ell}, u_{r}\right](\nu) & \text { if }\left(u_{\ell}, u_{r}\right) \in \mathcal{D}_{1}, \\
\left\{\begin{array}{ll}
\mathcal{R}_{\mathrm{PT}}\left[u_{\ell}, \hat{\mathrm{u}}\left(\mathrm{w}\left(u_{\ell}\right)\right)\right](\nu) & \text { if } \nu<0 \\
\mathcal{R}_{\mathrm{PT}}\left[\check{\mathrm{u}}\left(\mathrm{w}\left(u_{\ell}\right)\right), u_{r}\right](\nu) & \text { if } \nu \geqslant 0
\end{array} \text { if }\left(u_{\ell}, u_{r}\right) \in \mathcal{D}_{2} .\right.\end{cases}
$$

Remark 3.1. For any $u_{\ell}, u_{r} \in \Omega$ we have that $u(t, x) \doteq \mathcal{R}_{F}\left[u_{\ell}, u_{r}\right](x / t)$ has the following properties:

- Any discontinuity of u away from $x=0$ is classical, i.e., it satisfies the Lax entropy inequalities.

- Non-classical discontinuities of $u$ may occur only at $x=0$, and in this case the density flux at $x=0$ does not exceed the maximal flux $F$ allowed by the constraint. We stress that differently from the non-classical discontinuities performed by solutions of the constrained LWR model or of the constrained ARZ model, see [4,18], the non-classical shocks performed by solutions of constrained PT model (2) do not have density flux equal to $F$, see (5).

- Any discontinuity $\delta(t)$ of $x \mapsto u(t, x)$ satisfies the first Rankine-Hugoniot jump condition

$$
\left[\rho\left(t, \delta(t)_{+}\right)-\rho\left(t, \delta(t)_{-}\right)\right] \dot{\delta}(t)=f\left(u\left(t, \delta(t)_{+}\right)\right)-f\left(u\left(t, \delta(t)_{-}\right)\right),
$$

and if $\delta(t) \neq 0$, then it satisfies also the second Rankine-Hugoniot jump condition

$$
\begin{aligned}
& {\left[\rho\left(t, \delta(t)_{+}\right) \mathrm{W}\left(u\left(t, \delta(t)_{+}\right)\right)-\rho\left(t, \delta(t)_{-}\right) \mathrm{W}\left(u\left(t, \delta(t)_{-}\right)\right)\right] \dot{\delta}(t) } \\
= & f\left(u\left(t, \delta(t)_{+}\right)\right) \mathrm{W}\left(u\left(t, \delta(t)_{+}\right)\right)-f\left(u\left(t, \delta(t)_{-}\right)\right) \mathrm{W}\left(u\left(t, \delta(t)_{-}\right)\right) .
\end{aligned}
$$

The (extended) linearized momentum $\rho \mathrm{W}(u)$ is in general not conserved across non-classical shocks, even if they are between states in $\Omega_{\mathrm{c}}^{\mathrm{ex}}$. As a consequence, a solution to (2) taking values in $\Omega_{\mathrm{c}}^{\mathrm{ex}}$ is not necessarily a weak solution to ARZ model. This is in the same spirit of the solutions considered in [9, 20-23] for traffic through locations with reduced capacity.

\section{Blow-up example}

The following example shows that taking initial data in BV does not ensure the global existence of a solution to constrained Cauchy problem (2).
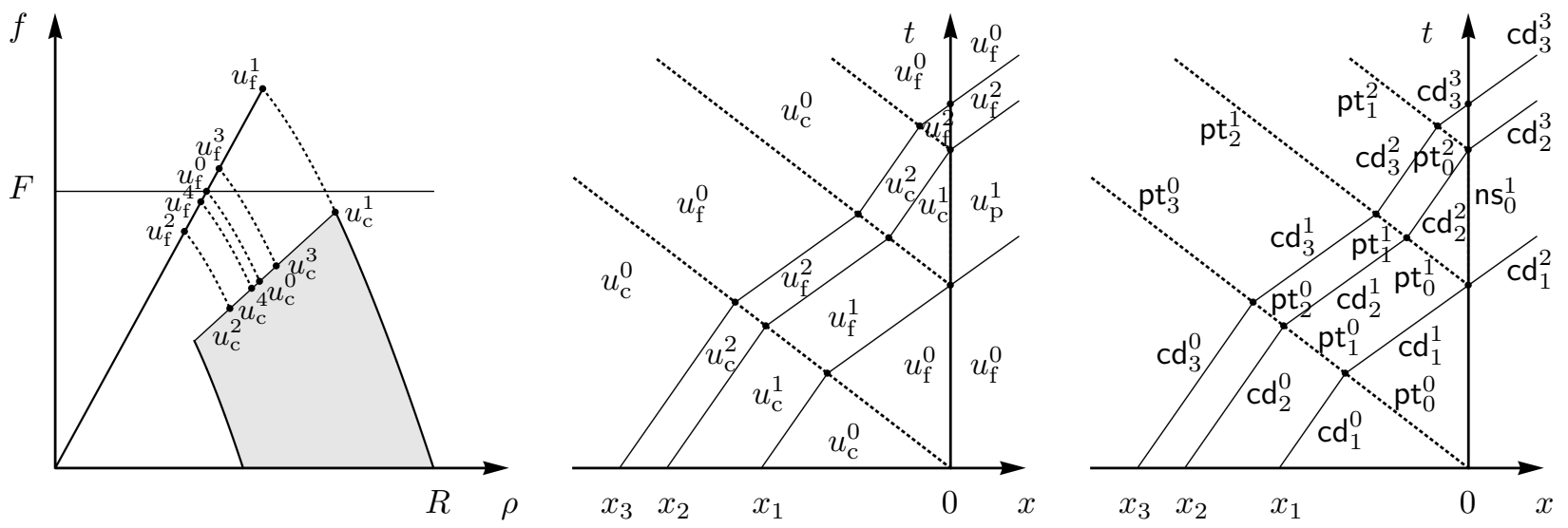

Figure 3: The approximate solution $u_{2}$. The dashed lines correspond to phase transitions.

Fix $F \in\left(f_{\mathrm{c}}^{+}, f_{\mathrm{f}}^{+}\right)$. Introduce

$$
u_{\mathrm{f}}^{0} \doteq u_{F} \in \Omega_{\mathrm{f}}^{+}, \quad u_{\mathrm{c}}^{0} \doteq \omega_{\mathrm{c}}\left(u_{F}\right) \in \Omega_{\mathrm{c}}, \quad u_{\mathrm{p}}^{0} \doteq\left(\frac{f\left(u_{\mathrm{c}}^{0}\right)}{V_{\mathrm{f}}}, V_{\mathrm{f}}\right) \in \Omega_{\mathrm{f}},
$$


and let $u_{\mathrm{f}}^{i} \in \Omega_{\mathrm{f}}^{+}, u_{\mathrm{c}}^{i} \in \Omega_{\mathrm{c}}, u_{\mathrm{p}}^{i} \in \Omega_{\mathrm{f}}, i \in \mathbb{N}$, be defined by

$$
\mathrm{w}\left(u_{\mathrm{c}}^{i}\right)=\mathrm{w}\left(u_{\mathrm{f}}^{i}\right)=\mathrm{w}\left(u_{\mathrm{f}}^{0}\right)+(-2)^{1-i} \mathfrak{w}, \quad v_{\mathrm{c}}^{i}=V_{\mathrm{c}}, \quad f\left(u_{\mathrm{p}}^{i}\right)=f\left(u_{\mathrm{c}}^{i}\right),
$$

where $\mathfrak{w} \doteq \min \left\{w^{+}-\mathrm{w}\left(u_{\mathrm{f}}^{0}\right), 2\left(\mathrm{w}\left(u_{\mathrm{f}}^{0}\right)-w^{-}\right)\right\}>0$. Clearly for any $i \in \mathbb{N}$ we have

$$
f\left(u_{\mathrm{f}}^{2 i}\right)<F=f\left(u_{\mathrm{f}}^{0}\right)<f\left(u_{\mathrm{f}}^{2 i-1}\right) .
$$

Let $x_{i} \doteq-1+2^{-i}, i \in \mathbb{N}$, and consider the initial datum

$$
u^{o}(x) \doteq \begin{cases}u_{\mathrm{f}}^{0} & \text { if } x>0, \\ u_{\mathrm{c}}^{0} & \text { if } x_{1}<x \leqslant 0, \\ u_{\mathrm{c}}^{i} & \text { if } x_{i+1}<x \leqslant x_{i}, \quad i \in \mathbb{N} \\ u_{\mathrm{c}}^{0} & \text { if } x \leqslant-1\end{cases}
$$

We approximate the initial datum with

$$
u_{n}^{o}(x) \doteq \begin{cases}u_{\mathrm{f}}^{0} & \text { if } x>0 \\ u_{\mathrm{c}}^{0} & \text { if } x_{1}<x \leqslant 0, \\ u_{\mathrm{c}}^{i} & \text { if } x_{i+1}<x \leqslant x_{i}, \quad i \in \mathbb{N} \cap[1, n] \\ u_{\mathrm{c}}^{0} & \text { if } x \leqslant x_{n+1} .\end{cases}
$$

Notice that $u^{o} \in \mathbf{B V}(\mathbb{R} ; \Omega)$ because $p^{-1}:(0, R) \rightarrow \mathbb{R}$ is Lipschitz by (1) and

$$
\operatorname{TV}\left(v^{o}\right)=V_{\mathrm{f}}-V_{\mathrm{c}}, \quad \operatorname{TV}\left(\mathrm{w}\left(u^{o}\right)\right)=\mathfrak{w}+\sum_{i=1}^{\infty} \frac{3}{2^{i}} \mathfrak{w}=4 \mathfrak{w}
$$

Furthermore we have

$$
\left\|u_{n}^{o}\right\|_{\mathbf{L}^{\infty}} \leqslant\left\|u^{o}\right\|_{\mathbf{L}^{\infty}}, \quad \operatorname{TV}\left(u_{n}^{o}\right) \leqslant \operatorname{TV}\left(u^{o}\right), \quad \lim _{n \rightarrow \infty}\left\|u_{n}^{o}-u^{o}\right\|_{\mathbf{L}^{\mathbf{1}}(\mathbb{R} ; \Omega)}=0 .
$$

We approximate the solution $u$ corresponding to the initial datum $u^{o}$ with the solution $u_{n}$ corresponding to the approximate initial datum $u_{n}^{o}$. Clearly $u_{n}$ is the juxtaposition of solutions to Riemann problems at the discontinuities of $u_{n}^{o}$ and at the interactions (between two waves or a wave and the interface $x=0$ ). We describe in detail only the case $n=2$, see Figure 3, the general case is analogous. Notice that any contact discontinuity $\left(u_{\mathrm{f}}^{h}, u_{\mathrm{f}}^{k}\right)$ has speed (of propagation) $V_{\mathrm{f}}$ and any contact discontinuity $\left(u_{\mathrm{c}}^{h}, u_{\mathrm{c}}^{k}\right)$ has speed $V_{\mathrm{c}}$.

- For times sufficiently small $u_{2}$ has the phase transition $\mathrm{pt}_{0}^{0} \doteq\left(u_{\mathrm{c}}^{0}, u_{\mathrm{f}}^{0}\right)$ with negative speed started at $x=0$, and three contact discontinuities $\mathrm{cd}_{1}^{0} \doteq\left(u_{\mathrm{c}}^{1}, u_{\mathrm{c}}^{0}\right), \mathrm{cd}_{2}^{0} \doteq\left(u_{\mathrm{c}}^{2}, u_{\mathrm{c}}^{1}\right)$ and $\mathrm{cd}_{3}^{0} \doteq\left(u_{\mathrm{c}}^{0}, u_{\mathrm{c}}^{2}\right)$ started at $x_{1}, x_{2}$ and $x_{3}$, respectively.

- The first interaction occurs between $\mathrm{cd}_{1}^{0}$ and $\mathrm{pt}_{0}^{0}$; the result is the phase transition $\mathrm{pt}_{1}^{0} \doteq\left(u_{\mathrm{c}}^{1}, u_{\mathrm{f}}^{1}\right)$ with negative speed and the contact discontinuity $\mathrm{cd}_{1}^{1} \doteq\left(u_{\mathrm{f}}^{1}, u_{\mathrm{f}}^{0}\right)$.

- When $\mathrm{cd}_{1}^{1}$ interacts with the interface $x=0$, the result is the phase transition $\mathrm{pt}_{0}^{1} \doteq\left(u_{\mathrm{f}}^{1}, u_{\mathrm{c}}^{1}\right)$ with negative speed, the stationary non-classical shock $\mathrm{ns}_{0}^{1} \doteq\left(u_{\mathrm{c}}^{1}, u_{\mathrm{p}}^{1}\right)$ and the contact discontinuity $\mathrm{cd}_{1}^{2} \doteq\left(u_{\mathrm{p}}^{1}, u_{\mathrm{f}}^{0}\right)$ with speed $V_{\mathrm{f}}$.

- When $\mathrm{cd}_{2}^{0}$ interacts with $\mathrm{pt}_{1}^{0}$, the result is the phase transition $\mathrm{pt}_{2}^{0} \doteq\left(u_{\mathrm{c}}^{2}, u_{\mathrm{f}}^{2}\right)$ with negative speed and the contact discontinuity $\mathrm{cd}_{2}^{1} \doteq\left(u_{\mathrm{f}}^{2}, u_{\mathrm{f}}^{1}\right)$.

- When $\mathrm{cd}_{2}^{1}$ interacts with $\mathrm{pt}_{0}^{1}$, the result is the phase transition $\mathrm{pt}_{1}^{1} \doteq\left(u_{\mathrm{f}}^{2}, u_{\mathrm{c}}^{2}\right)$ with negative speed and the contact discontinuity $\mathrm{cd}_{2}^{2} \doteq\left(u_{\mathrm{c}}^{2}, u_{\mathrm{c}}^{1}\right)$.

- When $\mathrm{cd}_{2}^{2}$ interacts with $\mathrm{ns}_{0}^{1}$, the result is the phase transition $\mathrm{pt}_{0}^{2} \doteq\left(u_{\mathrm{c}}^{2}, u_{\mathrm{f}}^{2}\right)$ with negative speed and the contact discontinuity $\mathrm{cd}_{2}^{3} \doteq\left(u_{\mathrm{f}}^{2}, u_{\mathrm{p}}^{1}\right)$.

- When $\mathrm{cd}_{3}^{0}$ interacts with $\mathrm{pt}_{2}^{0}$, the result is the phase transition $\mathrm{pt}_{3}^{0} \doteq\left(u_{\mathrm{c}}^{0}, u_{\mathrm{f}}^{0}\right)$ with negative speed and the contact discontinuity $\mathrm{cd}_{3}^{1} \doteq\left(u_{\mathrm{f}}^{0}, u_{\mathrm{f}}^{2}\right)$.

- When $\mathrm{cd}_{3}^{1}$ interacts with $\mathrm{pt}_{1}^{1}$, the result is the phase transition $\mathrm{pt}_{2}^{1} \doteq\left(u_{\mathrm{f}}^{0}, u_{\mathrm{c}}^{0}\right)$ with negative speed and the contact discontinuity $\mathrm{cd}_{3}^{2} \doteq\left(u_{\mathrm{c}}^{0}, u_{\mathrm{c}}^{2}\right)$. 
- When $\mathrm{cd}_{3}^{2}$ interacts with $\mathrm{pt}_{0}^{2}$, the result is the phase transition $\mathrm{pt}_{1}^{2} \doteq\left(u_{\mathrm{c}}^{0}, u_{\mathrm{f}}^{0}\right)$ with negative speed and the contact discontinuity $\mathrm{cd}_{3}^{3} \doteq\left(u_{\mathrm{f}}^{0}, u_{\mathrm{f}}^{2}\right)$.

- Finally $\mathrm{cd}_{3}^{3}$ crosses the interface $x=0$.

It is now clear how to construct $u_{n}$ for $n>2$. We point out that as $n$ grows, the number of contact discontinuities $\mathrm{cd}_{i}^{0} \doteq\left(u_{\mathrm{c}}^{i}, u_{\mathrm{c}}^{i-1}\right)$ appearing at $t=0$ increases. After some interactions, $\mathrm{cd}_{i}^{0}$ eventually evolves into a contact discontinuity $\mathrm{cd}_{i}^{i}$ that reaches the interface $x=0$ at some time $t=t_{i}$, creating in this way a phase transition $\mathrm{pt}_{0}^{i}$ with negative speed. Clearly $t_{i}<t_{i+1}<t_{n}<T_{\mathrm{c}} \doteq 1 / V_{\mathrm{c}}$ because any wave in $x<0$ moving with positive speed has speed (of propagation) bounded from below by $V_{\mathrm{c}}$ and starts at time $t=0$ at some $x \in(-1,0)$. Since the jump across each phase transition increases the total variation by at least $V_{\mathrm{f}}-V_{\mathrm{c}}$ and for any $t>T_{\mathrm{c}}$ the approximate solution $u_{n}(t, \cdot)$ contains at least $n+1$ phase transitions, we have that

$$
\operatorname{TV}\left(u_{n}\left(T_{\mathrm{c}}, \cdot\right)\right) \geqslant(n+1)\left(V_{\mathrm{f}}-V_{\mathrm{c}}\right) \text {. }
$$

As a consequence

$$
\lim _{n \rightarrow \infty} \operatorname{TV}\left(u_{n}\left(T_{\mathrm{c}}, \cdot\right)\right) \geqslant \lim _{n \rightarrow \infty}(n+1)\left(V_{\mathrm{f}}-V_{\mathrm{c}}\right)=\infty,
$$

thus the total variation of the solution $u$ explodes in a finite time.

\section{Acknowledgements}

The second author is member of GNAMPA. He acknowledges the support of the National Science Centre, Poland, Project "Mathematics of multi-scale approaches in life and social sciences" No. 2017/25/B/ST1/00051, of the INdAM-GNAMPA Project 2019 "Equazioni alle derivate parziali di tipo iperbolico o non locale ed applicazioni" and of University of Ferrara, FIR Project 2019 "Leggi di conservazione di tipo iperbolico: teoria ed applicazioni".

\section{References}

[1] Adimurthi, Ghoshal, S. S., and Veerappa Gowda, G. D. Structure of entropy solutions to scalar conservation laws with strictly convex flux. J. Hyperbolic Differ. Equ. 9, 4 (2012), 571-611.

[2] Ambrosio, L., And De Lellis, C. A note on admissible solutions of 1D scalar conservation laws and 2D Hamilton-Jacobi equations. J. Hyperbolic Differ. Equ. 1, 4 (2004), 813-826.

[3] Ambrosio, L., Fusco, N., and Pallara, D. Functions of bounded variation and free discontinuity problems. Oxford Mathematical Monographs. The Clarendon Press, Oxford University Press, New York, 2000.

[4] Andreianov, B. P., Donadello, C., Razafison, U., Rolland, J. Y., And Rosini, M. D. Solutions of the Aw-Rascle-Zhang system with point constraints. Networks and Heterogeneous Media 11, 1 (2016), 29-47.

[5] Aw, A., And Rascle, M. Resurrection of "second order" models of traffic flow. SIAM J. Appl. Math. 60, 3 (2000), 916-938 (electronic).

[6] Baiti, P., And Jenssen, H. K. Blowup in $L^{\infty}$ for a class of genuinely nonlinear hyperbolic systems of conservation laws. Discrete Contin. Dynam. Systems 7, 4 (2001), 837-853.

[7] Benyahia, M., Donadello, C., Dymski, N., and Rosini, M. D. An existence result for a constrained two-phase transition model with metastable phase for vehicular traffic. NoDEA Nonlinear Differential Equations Appl. 25, 5 (2018), Art. 48, 42.

[8] Benyahia, M., And Rosini, M. D. Entropy solutions for a traffic model with phase transitions. Nonlinear Analysis: Theory, Methods 83 Applications 141 (2016), 167 - 190.

[9] Benyahia, M., And Rosini, M. D. A macroscopic traffic model with phase transitions and local point constraints on the flow. Networks and Heterogeneous Media 12, 2 (2017), 297-317.

[10] Bianchini, S., And Caravenna, L. SBV regularity for genuinely nonlinear, strictly hyperbolic systems of conservation laws in one space dimension. Comm. Math. Phys. 313, 1 (2012), 1-33.

[11] Blandin, S., Work, D., Goatin, P., Piccoli, B., and Bayen, A. A general phase transition model for vehicular traffic. SIAM J. Appl. Math. 71, 1 (2011), 107-127.

[12] Bressan, A. Hyperbolic systems of conservation laws, vol. 20 of Oxford Lecture Series in Mathematics and its Applications. Oxford University Press, Oxford, 2000. The one-dimensional Cauchy problem.

[13] Bressan, A., Chen, G., And Zhang, Q. Lack of BV bounds for approximate solutions to the p-system with large data. J. Differential Equations 256, 8 (2014), 3067-3085.

[14] Bressan, A., And Colombo, R. M. Unique solutions of $2 \times 2$ conservation laws with large data. Indiana University Mathematics Journal 44, 3 (1995), 677-725.

[15] Bressan, A., And Colombo, R. M. Decay of positive waves in nonlinear systems of conservation laws. Ann. Scuola Norm. Sup. Pisa Cl. Sci. (4) 26, 1 (1998), 133-160.

[16] Caravenna, L., and Spinolo, L. V. Schaeffer's regularity theorem for scalar conservation laws does not extend to systems. Indiana Univ. Math. J. 66, 1 (2017), 101-160. 
[17] Colombo, R. M. Hyperbolic phase transitions in traffic flow. SIAM J. Appl. Math. 63, 2 (2002), 708-721 (electronic).

[18] Colombo, R. M., And Gontin, P. A well posed conservation law with a variable unilateral constraint. J. Differential Equations 234, 2 (2007), 654-675.

19] Colombo, R. M., Gontin, P., And Priuli, F. S. Global well posedness of traffic flow models with phase transitions. Nonlinear Anal. 66, 11 (2007), 2413-2426.

[20] Dal Santo, E., Rosini, M. D., Dymski, N., And Benyahia, M. General phase transition models for vehicular traffic with point constraints on the flow. Mathematical Methods in the Applied Sciences (2017), 1-19.

[21] Delle Monache, M. L., And Goatin, P. Stability estimates for scalar conservation laws with moving flux constraints. Netw. Heterog. Media 12, 2 (2017), 245-258.

[22] Dymski, N. S., Goatin, P., And Rosini, M. D. Existence of BV solutions for a non-conservative constrained Aw-Rascle-Zhang model for vehicular traffic. J. Math. Anal. Appl. 467, 1 (2018), 45-66.

[23] Garavello, M., and Goatin, P. The Aw-Rascle traffic model with locally constrained flow. Journal of Mathematical Analysis and Applications 378, 2 (2011), $634-648$.

[24] Glimm, J. Solutions in the large for nonlinear hyperbolic systems of equations. Comm. Pure Appl. Math. 18 (1965), $697-715$.

[25] Glimm, J., And Lax, P. D. Decay of solutions of systems of nonlinear hyperbolic conservation laws. Memoirs of the American Mathematical Society, No. 101. American Mathematical Society, Providence, R.I., 1970.

[26] Gontin, P. The Aw-Rascle vehicular traffic flow model with phase transitions. Mathematical and computer modelling 44, 3 (2006), 287-303.

[27] Holden, H., And Risebro, N. Front Tracking for Hyperbolic Conservation Laws. Applied Mathematical Sciences. Springer Berlin Heidelberg, 2013.

[28] Jenssen, H. K. Blowup for systems of conservation laws. SIAM J. Math. Anal. 31, 4 (2000), 894-908.

[29] Kruzhkov, S. N. First order quasilinear equations with several independent variables. Mat. Sb. (N.S.) 81 (123) (1970), $228-255$.

[30] LewickA, M. Well-posedness for hyperbolic systems of conservation laws with large BV data. Archive for Rational Mechanics and Analysis 173, 3 (Sep 2004), 415-445.

[31] Lighthill, M. J., And Whitham, G. B. On kinematic waves. II. A theory of traffic flow on long crowded roads. Proc. Roy. Soc. London. Ser. A. 229 (1955), 317-345.

[32] LiU, T. P. Decay to $N$-waves of solutions of general systems of nonlinear hyperbolic conservation laws. Comm. Pure Appl. Math. 30, 5 (1977), 586-611.

[33] Oleinik, O. A. Discontinuous solutions of non-linear differential equations. Uspehi Mat. Nauk (N.S.) 12, 3(75) (1957), 3-73.

[34] Richards, P. I. Shock waves on the highway. Operations Res. 4 (1956), 42-51.

[35] Rosini, M. D. Macroscopic models for vehicular flows and crowd dynamics: theory and applications. Understanding Complex Systems. Springer, Heidelberg, 2013.

[36] Schaeffer, D. G. A regularity theorem for conservation laws. Advances in Math. 11 (1973), $368-386$.

[37] Zhang, H. M. A non-equilibrium traffic model devoid of gas-like behavior. Transportation Research Part B: Methodological 36,3 (2002), 275-290. 